\title{
Arcuate neuropeptide $Y$ inhibits sympathetic nerve activity via multiple neuropathways
}

\author{
Zhigang Shi, ${ }^{1}$ Christopher J. Madden, ${ }^{2}$ and Virginia L. Brooks ${ }^{1}$ \\ 'Department of Physiology and Pharmacology and 2Department of Neurological Surgery, Oregon Health \& Science University, Portland, Oregon, USA.
}

\begin{abstract}
Obesity increases sympathetic nerve activity (SNA) via activation of proopiomelanocortin neurons in the arcuate nucleus (ArcN), and this action requires simultaneous withdrawal of tonic neuropeptide Y (NPY) sympathoinhibition. However, the sites and neurocircuitry by which NPY decreases SNA are unclear. Here, using designer receptors exclusively activated by designer drugs (DREADDs) to selectively activate or inhibit ArcN NPY neurons expressing agouti-related peptide (AgRP) in mice, we have demonstrated that this neuronal population tonically suppresses splanchnic SNA (SSNA), arterial pressure, and heart rate via projections to the paraventricular nucleus (PVN) and dorsomedial hypothalamus (DMH). First, we found that ArcN NPY/AgRP fibers closely appose PVN and DMH presympathetic neurons. Second, nanoinjections of NPY or an NPY receptor Y1 (NPY1R) antagonist into PVN or DMH decreased or increased SSNA, respectively. Third, blockade of DMH NPY1R reversed the sympathoinhibition elicited by selective, DREADD-mediated activation of ArcN NPY/AgRP neurons. Finally, stimulation of ArcN NPY/AgRP terminal fields in the PVN and DMH decreased SSNA. Considering that chronic obesity decreases ArcN NPY content, we propose that the ArcN NPY neuropathway to the PVN and DMH is pivotal in obesity-induced elevations in SNA.
\end{abstract}

\section{Introduction}

Obesity increases the risk of hypertension, secondary to activation of the sympathetic nervous system $(1,2)$. However, the cellular molecular mechanisms in brain that link increases in adiposity with elevations in sympathetic nerve activity (SNA) remain unclear. Proopiomelanocortin (POMC) neurons in the arcuate nucleus (ArcN) are key contributors (3). However, POMC-induced sympathoexcitation requires simultaneous withdrawal of tonic neuropeptide Y (NPY) inhibition $(4,5)$. Thus, NPY acts as a critical gatekeeper for the excitatory inputs that drive sympathoexcitation, suggesting that suppressed NPY inhibition must occur in parallel to increased POMC excitation with obesity. Indeed, obesity decreases hypothalamic NPY content (6). However, whether hypothalamic NPY contributes to obesity-induced increases in SNA has not been investigated.

A major impediment to understanding the role of NPY in obesity-induced sympathoexcitation is that the brain sites and pathways by which NPY reduces SNA have only begun to be explored. In rats, the hypothalamic paraventricular nucleus (PVN) is a major site of action, at which NPY directly inhibits presympathetic neurons that project to the rostral ventrolateral medulla (RVLM), which drives basal sympathetic tone (4). Nevertheless, the sources of NPY inputs to PVN that elicit sympathoinhibition have not been identified. Indirect evidence implicates an NPY projection from the ArcN. More specifically, injections of leptin or insulin into the $\operatorname{ArcN}$, or nonspecific activation of the ArcN, increase SNA in part via suppres-

Conflict of interest: The authors have declared that no conflict of interest exits. Submitted: November 29, 2016; Accepted: April 27, 2017

Reference information: J Clin Invest. 2017;127(7):2868-2880.

https://doi.org/10.1172/JCI92008. sion of tonic NPY inhibition of PVN presympathetic neurons $(5,7,8)$. However, whether the PVN NPY input that is suppressed by insulin and leptin originates directly from neurons in the ArcN has not been established. In addition to the ArcN, (9), NPY projections from the brainstem (10) and, under some circumstances, the dorsomedial hypothalamus (DMH) (11) are alternative candidates. Moreover, whether other ArcN NPY projections influence the sympathetic nervous system or arterial pressure (AP) has not been investigated. Therefore, the present study was undertaken to test the hypothesis that ArcN NPY neurons decrease SNA via several projections, including the PVN. This information is critical, since derangements in brain NPY expression and activity are known to occur in several states that increase AP, ranging from obesity to anxiety $(6,12)$. Moreover, NPY agonists and antagonists are being avidly explored as therapeutic agents in these and other conditions (13).

\section{Results}

Selective activation or inhibition of ArcN NPY/AgRP neurons decreases and increases $S N A$, respectively. The recently developed chemogenetic approach, in which designer receptors are exclusively activated by the designer drug (DREADD) clozapine-N-oxide (CNO), has proven powerful in delineating the neurocircuitry by which $\mathrm{ArcN}$ NPY neurons stimulate food intake $(14,15)$. Nearly all ArcN NPY neurons also express agouti-related peptide (AgRP) (16). Therefore, the excitatory DREADD hM3Dq or the inhibitory DREADD hM4Di was selectively expressed in ArcN NPY/AgRP neurons by nanoinjecting a Cre-dependent adeno-associated virus (AAV) carrying a DREADD and a mCherry reporter into the ArcN of AgrpIRES-Cre mice (ArcN hM3Dq mice and ArcN hM4Di mice). To confirm the specificity of hM3Dq expression in NPY neurons, we first nanoinjected AAV-hM3Dq-mCherry into mice that expressed 
A
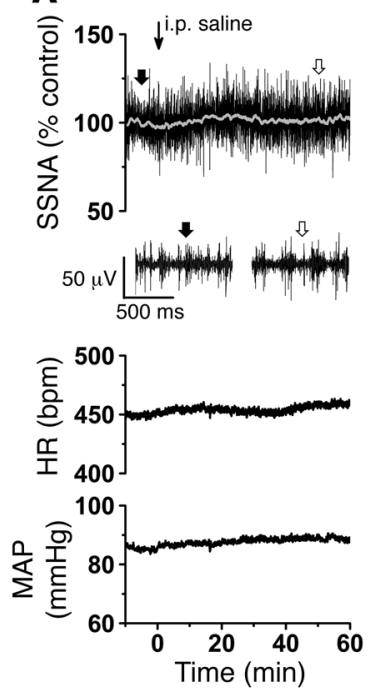

- WT + saline $(n=7)$
B
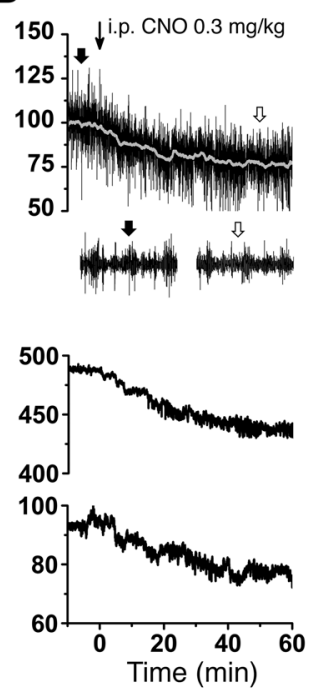

C
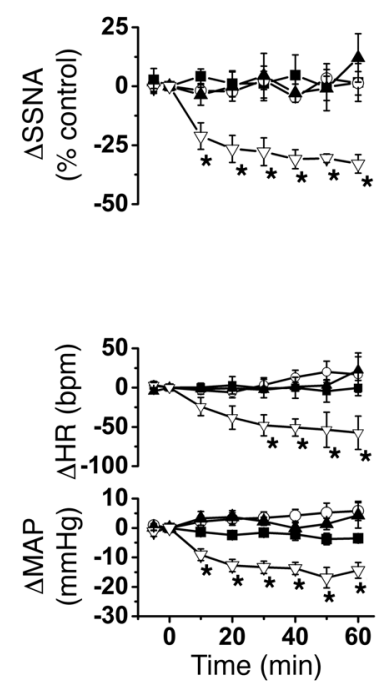

$\nabla \mathrm{hM} 3 \mathrm{Dq}+\mathrm{CNO}(n=7)$
D
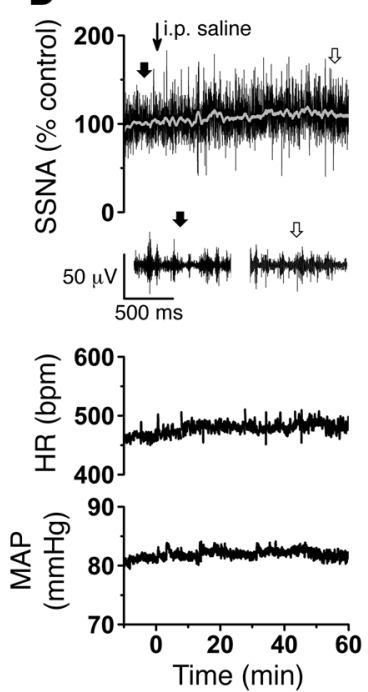

E
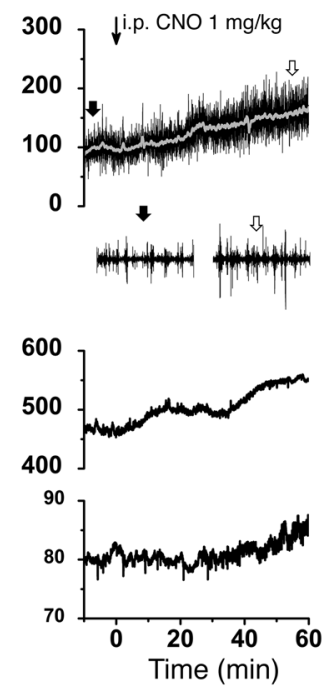

$\mathbf{F}$
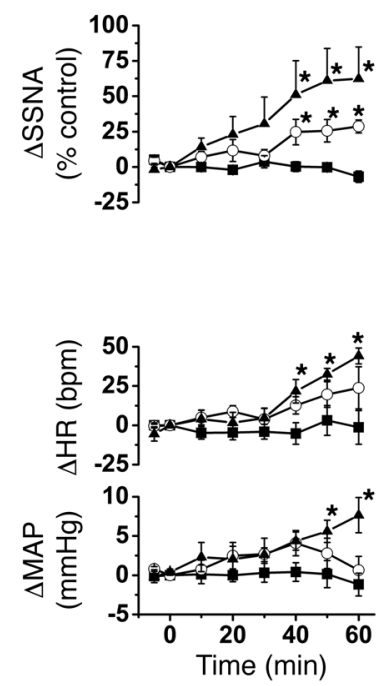

- $\mathrm{hM} 4 \mathrm{Di}+$ saline $(n=6) \quad \mathrm{O} \mathrm{hM} 4 \mathrm{Di}+\mathrm{CNO} 0.3 \mathrm{mg} / \mathrm{kg}(n=5)$

- $\mathrm{hM} 4 \mathrm{Di}+\mathrm{CNO} 1 \mathrm{mg} / \mathrm{kg}(n=7)$

Figure 1. Selective activation and inhibition of ArcN NPY/AgRP neurons decreases and increases SNA, respectively. (A) Representative experiment showing that i.p. injection of saline (at thin arrow) has no effect on raw SSNA, HR, and MAP in an ArcN hM3Dq mouse. Expanded raw nerve tracings, before and after injection, are indicated in this and all subsequent figures by filled and open arrows, respectively. (B) Representative experiment showing that i.p. injection of CNO (at thin arrow) in an ArcN hM3Dq mouse immediately decreases raw SSNA, HR, and MAP. (C) Grouped data showing that i.p. CNO, but not saline, decreases SSNA, HR, and MAP in ArcN hM3Dq mice. Neither saline nor CNO had effects in WT mice that received hM3Dq. Baseline values in WT mice $(n=13)$ were $88 \pm 3 \mathrm{mmHg}$ and 469 $\pm 13 \mathrm{bpm}$ and in Agrp-IRES-Cre $(n=12)$ were $91 \pm$ $3 \mathrm{mmHg}$ and $460 \pm 16 \mathrm{bpm}$. (D). Representative experiment showing that i.p. injection of saline (at thin arrow) has no effect on raw SSNA, HR, and MAP in an ArcN hM4Di mouse. (E) Representative experiment showing that i.p. injection of CNO (at thin arrow) in an ArcN hM4Di mouse increases raw SSNA, HR, and MAP. (F). Grouped data showing that i.p. CNO, but not saline, dose-dependently increases SSNA, HR, and MAP in ArcN hM4Di mice. Baseline values were $78 \pm$ $3 \mathrm{mmHg}$ and $466 \pm 14 \mathrm{bpm}(n=11) .{ }^{*} P<0.05$, compared with time 0 . both NPY-GFP and Cre in AgRP neurons. In agreement with an earlier study using a different approach (14), we found that while not all NPY-GFP neurons had visible hM3Dq-mCherry labeling, $100 \%$ of the hM3Dq-mCherry-marked neurons also expressed NPY-GFP (Supplemental Figure 1; supplemental material available online with this article; https://doi.org/10.1172/JCI92008DS1). WT mice never expressed the Cre-dependent DREADD. In a second set of experiments, 2 or more weeks after Agrp-IRES-Cre mice received hM3Dq bilaterally in the $\operatorname{ArcN}, \mathrm{CNO}(0.3 \mathrm{mg} / \mathrm{kg})$ or the saline vehicle was administered i.p., and food intake was monitored for the following 4 hours. As shown previously (14), i.p. CNO, but not saline, rapidly evoked food intake in ArcN hM3Dq mice (Supplemental Figure 1). Conversely, activation of inhibitory DREADDs in ArcN hM4Di mice at the beginning of the feeding period (lights out) inhibited food intake (Supplemental Figure 1). Therefore, we anatomically and functionally confirm that this approach selectively targets ArcN NPY/AgRP neurons.
We next tested whether selective excitation or inhibition of ArcN NPY/AgRP neurons alters splanchnic SNA (SSNA), mean AP (MAP), or heart rate (HR) (Figure 1). As shown in representative experiments (Figure 1, A and $\mathrm{B}$ ) and grouped data (Figure $1 \mathrm{C}$ ), in ArcN hM3Dq mice, i.p. CNO $(0.3 \mathrm{mg} / \mathrm{kg})$ promptly decreased SSNA, MAP, and HR, whereas i.p. saline had no effects. The suppression induced by CNO was sustained for at least 1 hour, and, in mice with longer recordings, up to 4 hours (data not shown). In rats, guinea pigs, and humans, CNO can have nonspecific effects due to its conversion to clozapine $(17,18)$. However, in WT mice receiving the Cre-dependent hM3Dq vector, neither i.p. saline nor CNO significantly altered these variables, suggesting that $\mathrm{CNO}$ was not exerting its effects independently of DREADD activation. A separate group of Agrp-IRES-Cre mice instead received AAV-hM4Di-mCherry in the ArcN (Figure 1, D-F). In these ArcN hM4Di mice, i.p. CNO (0.3 and $1 \mathrm{mg} / \mathrm{kg}$ ) dose-dependently increased SSNA, MAP, and HR, although the responses developed 

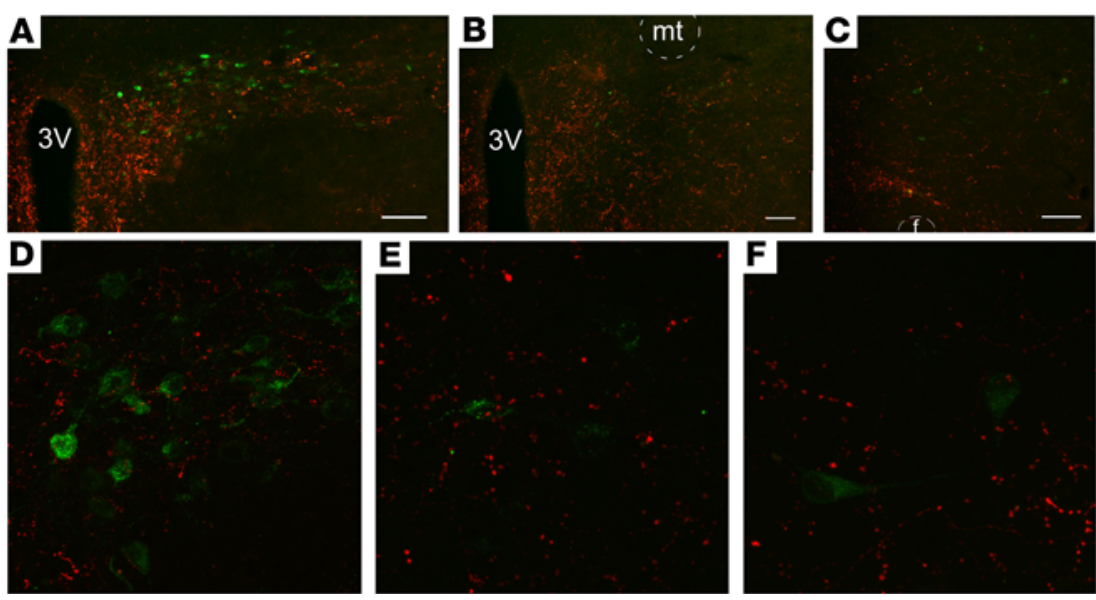

Figure 2. Neurons in the PVN, DMH, and LH that project to the RVLM appear to receive inputs from ArcN NPY/AgRP neurons. CtB-immunoreactive neurons (green) and Ds-red-immunoreactive fibers and terminals in the PVN (A; $52 \pm 11$ CtB cells, $n=3$ ), DMH (B; $41 \pm 18$ CtB cells, $n=3$ ), and LH (C; $54 \pm 26$ CtB cells, $n=3$ ) following injection of CtB in the RVLM (injection sites illustrated in Supplemental Figure 2) and synaptically directed Cre-dependent mCherry expression in ArcN NPY/AgRP neurons. Scale bars: $100 \mu \mathrm{m}$. (D) Confocal image of a single plane $\left(192 \mu \mathrm{m}^{2}\right)$ illustrating that several PVN neurons that are retrogradely labeled by CtB (green) receive close appositions from ArcN NPY/AgRP fibers and terminals (red). (E and F) Confocal images of single planes (each $112 \mu \mathrm{m}^{2}$ ) illustrating that several scattered CtB-labeled neurons were also observed in the DMH (E) and the LH (F). In the DMH, some of RVLM-projecting neurons also received $\operatorname{ArcN}$ NPY/AgRP appositions; however, in the LH these appositions were rare. These images are representative of the results from the 3 mice in which CtB injections encompassed the RVLM. $\mathrm{BV}$, third ventricle; $\mathrm{f}$, fornix; $\mathrm{mt}$, mamillothalamic tract.

more slowly than in ArcN hM3Dq mice and the increases in MAP were delayed compared to the increases in SSNA and HR. Again, i.p. saline had no significant effects. From these data we conclude that ArcN NPY/AgRP neurons can suppress SSNA, AP, and HR. Moreover, these data suggest that this neuronal population tonically restrains SNA and AP.

$R V L M$-projecting neurons in the $P V N$ and the $D M H$ receive $A r c N$ $N P Y / A g R P$ inputs, whereas in the LH, NPY/AgRP fibers only occasionally appose RVLM-projecting cell bodies. We next sought to delineate the projection targets of ArcN NPY/AgRP neurons that mediate sympathoinhibition when these neurons are stimulated by excitatory DREADD activation in ArcN hM3Dq mice. ArcN NPY/AgRP neurons innervate many hypothalamic sites that influence SNA and AP, most extensively the PVN, the $\mathrm{DMH}$, and the lateral hypothalamus/perifornical nucleus $(\mathrm{LH})(9,16,19)$. However, whether RVLM-projecting neurons reside in these hypothalamic nuclei in mice or whether these neurons are innervated by ArcN NPY/AgRP terminal fields is unknown. Therefore, we identified presympathetic neurons by injecting the retrogradely transported indicator cholera toxin B (CtB) into the RVLM (Supplemental Figure 2). We then determined, using confocal microscopy, whether PVN, DMH, or LH CtB-labeled neurons are closely associated with ArcN NPY/ AgRP neuronal terminals, identified using a Cre-dependent synaptically targeted mCherry AAV injected bilaterally into the ArcN of Agrp-IRES-Cre mice. We observed numerous PVN neurons that project to the RVLM (Figure 2). In addition, a substantial fraction of these neurons exhibited adjacent ArcN NPY/AgRP fibers (Figure $2 ; 36 \% \pm 4 \%$ of $\mathrm{CtB}$-labeled neurons received a close apposition from ArcN NPY/AgRP fibers; $n=3$. Several scattered CtB-labeled neurons were also observed in the $\mathrm{DMH}$ and LH (Figure 2). In the DMH, $31 \% \pm 10 \%$ of the CtB-labeled neurons were closely associated with ArcN NPY/AgRP fibers. In contrast, in the LH, ArcN NPY/AgRP fibers only occasionally apposed CtB-positive cell bodies (Figure 2, $6 \pm$ 3 cells; $14 \% \pm 8 \%)$. Other brain sites that exhibited several CtB-positive (RVLM-projecting) neurons and mCherry ArcN NPY/AgRP fibers were the preoptic area (POA), the ventrolateral periaqueductal gray (vlPAG), and the lateral parabrachial nucleus (LPB) (Supplemental Figure 3). Therefore, we conclude that in mice, the PVN, DMH, LH, POA, vlPAG, and LPB contain presympathetic neurons that project to the RVLM. Moreover, of the major projection targets of ArcN NPY/AgRP neurons (PVN, DMH, LH), both the PVN and DMH house a substantial number of RVLM-projecting cells that receive inputs from ArcN NPY/AgRP neurons. Nevertheless, given that peptides like NPY can signal via longer-range volume transmission $(20,21)$, it remains possible that ArcN NPY inputs can also influence the activity of other RVLM-projecting neurons, even those that lack a close apposition.

NPY suppresses SNA, MAP, and HR in the $P V N$ and DMH, but not the LH. Since it is currently unknown in mice whether NPY inhibits SNA by binding to receptors in the PVN, DMH, or LH, we next tested the effects of bilateral nanoinjections (30 $\mathrm{nl})$ of artificial CSF (aCSF) vehicle or NPY at a dose that decreases SNA in rats (4). In rats, PVN NPY-induced sympathoinhibition is transduced via NPY Y1 receptors (NPY1R) and, to a lesser extent, NPY Y5 receptors (NPY5R) (4). However, it appears that NPY5R receptors are not expressed in the mouse PVN $(22,23)$. Therefore, we also tested the actions of the highly selective NPY1R antagonist BIBO3304 at a dose higher than that used in rats (4) but lower than the dose used to block feeding via PVN injection in mice (24). In the PVN (Figure 3), NPY decreased SSNA and MAP, without significantly altering $\mathrm{HR}$, whereas BIBO3304 increased SSNA, MAP, and HR. These variables were unaffected by PVN aCSF injections. In the DMH (Figure 3), NPY decreased SSNA, MAP, and HR, and BIBO3304 increased SSNA, MAP, and HR. However, compared with the PVN, the increases in MAP and HR elicited by blockade of DMH NPY1R were nearly twice as great $(P<0.05)$, and the increase in SSNA tended to be larger $(P=0.08)$. Importantly, injections just outside of the PVN or DMH failed to significantly alter SSNA, MAP, or HR (Supplemental Figure 4), indicating that these responses were selective to these hypothalamic sites. In contrast to the DMH and PVN, while increases and decreases in SSNA were observed, overall neither NPY nor BIBO3304 significantly changed SSNA, HR, and MAP when injected into the LH (Supplemental Figure 5). Thus, as in rats (4), NPY inhibits SNA via an action in the PVN in mice. We further identify the DMH as an additional site of action. Finally, these data indicate that NPY released in the PVN and DMH could mediate the decreases in SNA evoked by hM3Dq stimulation of ArcN NPY/AgRP neurons. 
A

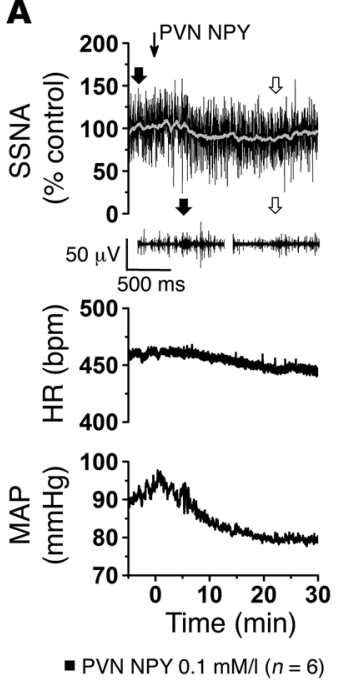

E
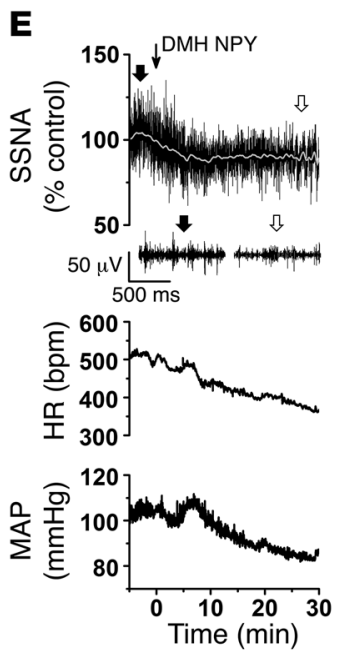

- DMH NPY $0.1 \mathrm{mM} / \mathrm{I}(n=7)$
B
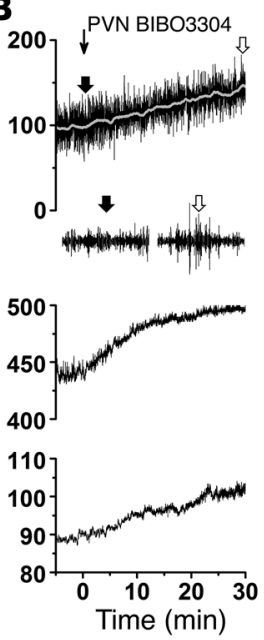

O PVN aCSF $(n=7)$
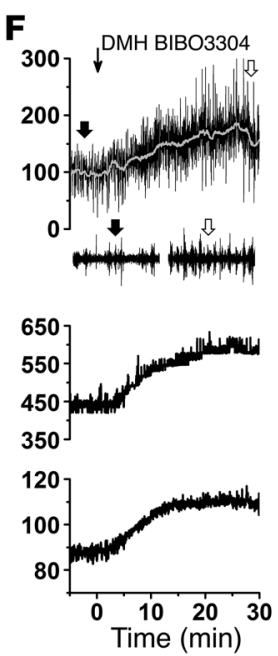

oDMH aCSF $(n=7)$

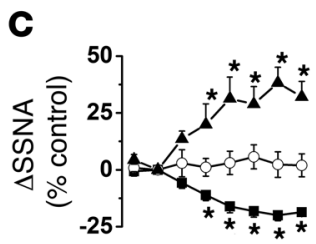

D
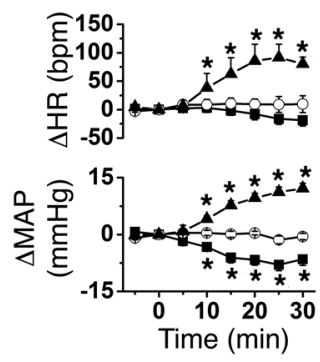

\ PVN BIBO3304 $10 \mathrm{mM} / \mathrm{l}(n=6)$

\section{G}
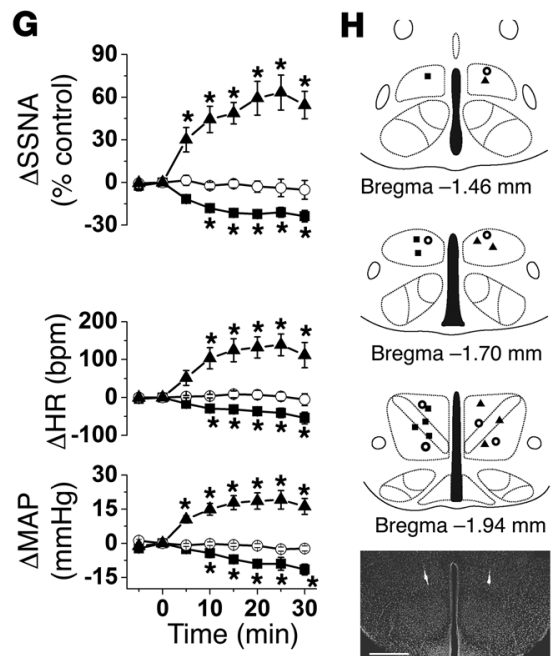

^ DMH BIBO3304 $10 \mathrm{mM} / \mathrm{l}(n=6)$
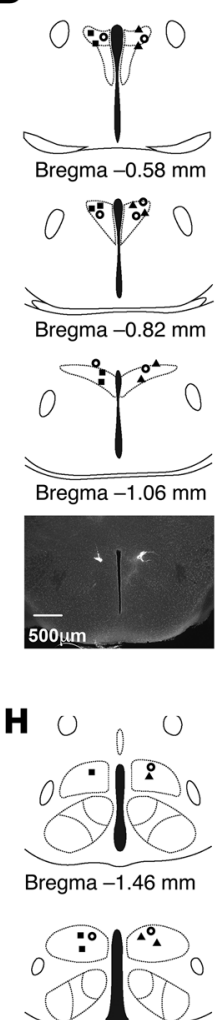

Bregma $-1.70 \mathrm{~mm}$

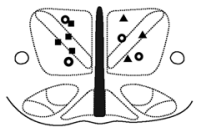

Bregma $-1.94 \mathrm{~mm}$

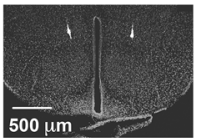

Figure 3. Bilateral nanoinjections of NPY or BIBO3304 into the PVN and DMH of WT mice decrease and increase SSNA, HR, and MAP, respectively. (A and E). Representative experiments showing that injections (at thin arrow) of NPY into the PVN or DMH decrease raw SSNA, HR, and MAP. (B and F) Representative experiments showing that injections (at thin arrow) of BIBO3304 into the PVN or DMH increase raw SSNA, HR and MAP. (C) Grouped data showing that PVN NPY or BIB03304 decreases or increases SSNA, HR, and MAP, respectively; PVN injections of aCSF had no effects. Baseline values were $88 \pm 3 \mathrm{mmHg}$ and $443 \pm 15 \mathrm{bpm}$ $(n=19)$. (G) Grouped data showing that $\mathrm{DMH}$ injections of NPY or BIBO3304 decrease or increase SSNA, HR, and MAP, respectively; DMH injections of aCSF had no effects. Baseline values were $86 \pm 2 \mathrm{mmHg}$ and 450 $\pm 12 \mathrm{bpm}(n=20)$. (D and $\mathbf{H})$ Histological maps (based on ref. 80), and representative sections illustrating PVN and DMH injection sites. ${ }^{*} P<0.05$, compared with time 0 .
The PVN and DMH are targets of ArcN NPY/AgRP sympathoinhibitory neurons. To test the involvement of these nuclei, we first nanoinjected BIBO3304 bilaterally into the PVN or DMH of ArcN hM3Dq mice, $~ 30-45$ minutes after i.p. CNO injection (i.e., when responses were stable). Our rationale was the following: if PVN or DMH NPY1R is a major component of the inhibition evoked by ArcN AgRP/NPY neuronal hM3Dq activation, then BIBO3304 should not only reverse the decreases in SSNA, MAP, and HR elicited by CNO, but also elevate these variables to the same levels achieved following PVN or DMH BIBO3304 treatment in i.p. saline-injected animals (i.e., block both tonic basal NPY release and CNO-triggered release). In ArcN hM3Dq mice that received i.p. saline, PVN BIBO3304 increased SSNA, MAP, and HR (Figure 4) to levels similar to those observed in WT mice (Figure 3), indicating that ArcN AAV injections did not alter tonic NPY inhibition of PVN presympathetic neurons. As in Figure 1, i.p. injection of CNO in ArcN hM3Dq mice again decreased SSNA, MAP, and $\mathrm{HR}$, and these responses were sustained in mice that received PVN aCSF (Figure 4). However, while subsequent PVN BIBO3304 significantly increased SSNA, MAP, and HR, the levels remained lower than those achieved in mice injected first with i.p. saline and then with PVN BIBO3304 (Figure 4). A potential concern is that after $\mathrm{CNO}$ pretreatment, nonspecific effects of $\mathrm{CNO}$ prevented BIBO3304 from increasing SSNA, MAP, and HR to the same extent as BIBO3304 administered after saline pretreatment. However, in WT mice that received the Cre-dependent hM3Dq vector in the ArcN, i.p. CNO had no effects ( $\triangle \mathrm{SSNA}$ : $1 \% \pm 6 \% ; \triangle \mathrm{MAP}$ : $-3 \pm 1 \mathrm{mmHg}$; HR: $2 \pm 13 \mathrm{bpm} ; n=4$ ), and subsequent PVN injections of BIBO3304 increased these variables to the same extent as in untreated WT mice ( $\triangle$ SSNA: to $33 \% \pm 3 \%$ control; $\triangle \mathrm{MAP}$ : $9 \pm 2 \mathrm{mmHg}$; $\triangle \mathrm{HR}$ : $66 \pm 17 \mathrm{bpm}$ ). In the $\mathrm{DMH}$, however, bilateral BIBO3304 not only reversed the decreases in SSNA, MAP, and HR evoked by CNO in ArcN hM3Dq mice, but also elevated these variables to values that were not different from those observed in mice given i.p. saline followed by DMH BIBO3304. Therefore, we conclude that NPY1R activation in the DMH is a major contributor to the decreases in SSNA, MAP, and HR following selective activation of ArcN NPY neurons.

Our anatomical studies revealed that numerous caudal PVN neurons that target the RVLM appear heavily innervated by ArcN NPY/AgRP terminals (Figure 2). Moreover, PVN neurons are known to project directly to sympathetic preganglionic neu- 
A

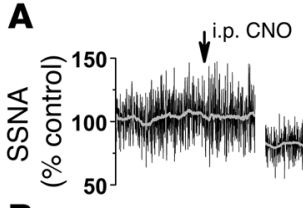

\section{焉}
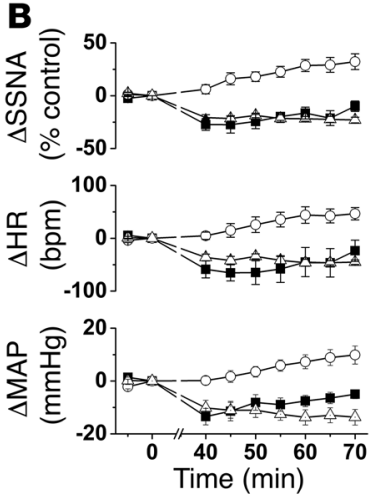

E
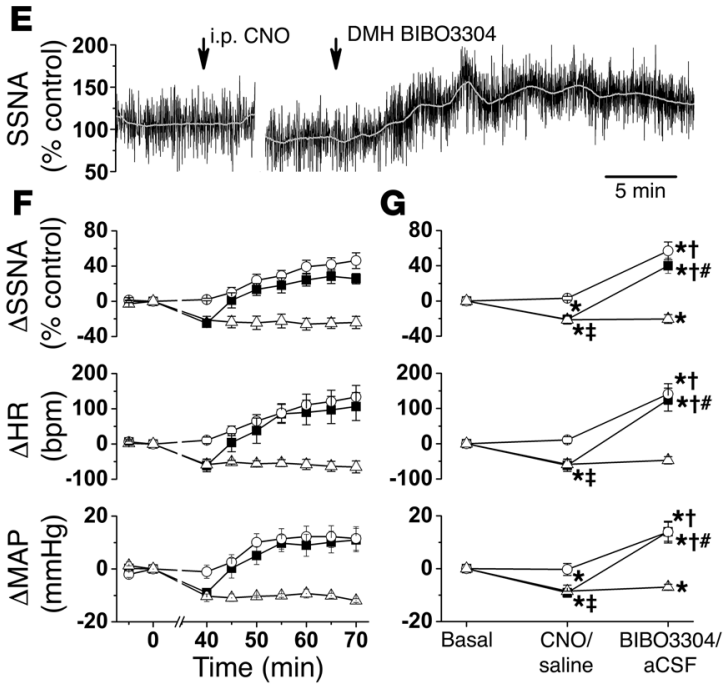

D
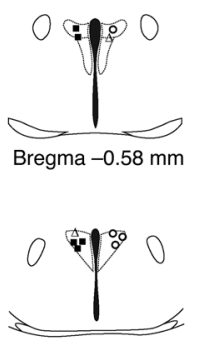

Bregma $-0.82 \mathrm{~mm}$
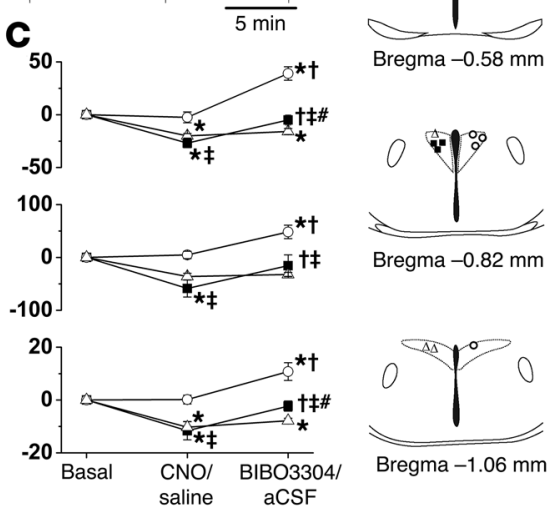

- Saline + PVN BIBO3304 $(n=5) \quad \triangle \mathrm{CNO}+\operatorname{PVN}$ aCSF $(n=4)$

H

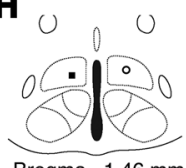

Bregma $-1.46 \mathrm{~mm}$
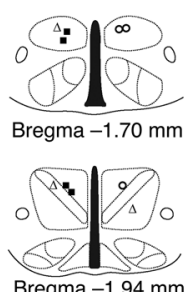

Bregma $-1.94 \mathrm{~mm}$

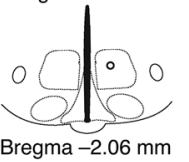

- $\mathrm{CNO}+\mathrm{DMH} \mathrm{BIBO} 304(n=5) \quad \circ$ Saline + DMH BIBO3304 $(n=5) \quad \triangle \mathrm{CNO}+\mathrm{DMH}$ aCSF $(n=3)$

rons in the spinal cord (25). Therefore, given the profound sympathoinhibitory effects of NPY in the PVN in both mice (Figure 3 ) and rats $(4,7,26)$, we next considered potential explanations for the failure of PVN BIBO3304 administered after i.p. CNO to achieve SSNA levels similar to those elicited by PVN BIBO3304 after i.p. saline. First, we tested whether our BIBO3304 dose both reverses the effects of exogenous NPY in PVN and elevates SSNA, MAP, and HR above basal levels by reversing the actions of tonic endogenous NPY sympathoinhibition. This result was obtained (Supplemental Figure 6), indicating that the BIBO3304 dose is sufficient to produce the expected effects if the PVN is a major target of ArcN NPY projections. ArcN NPY/AgRP neurons also release AgRP and GABA; therefore, we next tested whether bilateral nanoinjections of AgRP and muscimol $\left(\mathrm{GABA}_{\mathrm{A}}\right.$ receptor agonist) would mimic (or simulate)the actions of these released neurotransmitters after ArcN NPY/AgRP neuronal activation. We chose a dose of AgRP that is sufficient to stimulate food intake in mice when administered into the cerebroventricles $(27,28)$. However, when locally injected into the PVN, AgRP had no effects on
Figure 4. Bilateral nanoinjection of BIB03304 into the DMH, but not the PVN, reverses the effects of select activation of ArcN NPY/AgRP neurons. (A) Representative experiment in an ArcN hM3Dq mouse showing that PVN BIB03304 increases SSNA after i.p. CNO. (B and $\mathbf{C}$ ) Grouped data illustrate the time course of changes in SSNA, HR, and MAP (B), and the results of 2-way ANOVA (C) show that i.p. CNO, but not i.p. saline, significantly decreases SSNA, HR, and MAP. Subsequent PVN BIB03304 increased these variables in both groups; however, SSNA, HR, and MAP in mice given i.p. CNO remained below values obtained in mice given i.p. saline. On the other hand, the responses to i.p. CNO remained stable after PVN aCSF injections. Baseline values were $89 \pm 3 \mathrm{mmHg}$ and $486 \pm 12 \mathrm{bpm}(n=15)$. (D) Histological maps illustrating PVN injection sites. (E) Representative experiment showing that DMH BIB03304 increases SSNA after i.p. CNO in an ArcN hM3Dq mouse. ( $F$ and $\mathbf{G}$ ) Grouped data illustrate the time course of changes in SSNA, HR, and MAP (F), and the results of 2-way repeated-measures ANOVA (C) show that i.p. CNO, but not i.p. saline, significantly decreased SSNA, HR, and MAP; subsequent DMH BIB03304 significantly increased these variables to the same levels in both groups. On the other hand, the responses to i.p. CNO remained stable after DMH aCSF injections. Baseline values were $88 \pm 3 \mathrm{mmHg}$ and $456 \pm 14 \mathrm{bpm}$ $(n=13)$. (H) Histological maps illustrating DMH injection sites. ${ }^{*} P$ $<0.05$, compared with time $0 ;{ }^{\dagger} P<0.05$ compared with previous value within group; ${ }^{\ddagger} P<0.05$ between groups receiving i.p. saline versus i.p. CNO and PVN/DMH BIB03304; ${ }^{*} P<0.05$ between groups receiving i.p. CNO and PVN/DMH aCSF versus i.p. CNO and PVN/DMH BIB03304.
SSNA, MAP, or HR, despite triggering a significant decrease in body temperature (Supplemental Figure 7). The dose of muscimol potently reverses increases in SNA when nanoinjected into the PVN of rats $(5,29)$ and is sufficient to inhibit behavior when nanoinjected in the mouse forebrain $(30,31)$. Likewise, PVN muscimol did not reduce SSNA or HR, but did decrease MAP (Supplemental Figure 8). Because hypothalamic nanoinjections of muscimol coupled with sympathetic nerve recordings have not been conducted previously in mice, for comparison, we also injected muscimol into the $\mathrm{DMH}$, after which the agonist slightly decreased HR and MAP, but not SSNA (Supplemental Figure 8). Conversely, blockade of PVN or DMH GABA ${ }_{A}$ receptors with gabazine elicited robust increases in SSNA, HR, and MAP (Supplemental Figure 9). Interestingly, gabazine had opposite effects on body temperature in the PVN and DMH, as expected from experiments in rats $(32,33)$, which also confirms the site selectivity of the injections. Collectively these data suggest that neither AgRP nor GABA in PVN are involved in the suppression of SSNA or HR following ArcN AgRP/NPY neuronal activation, likely because of 

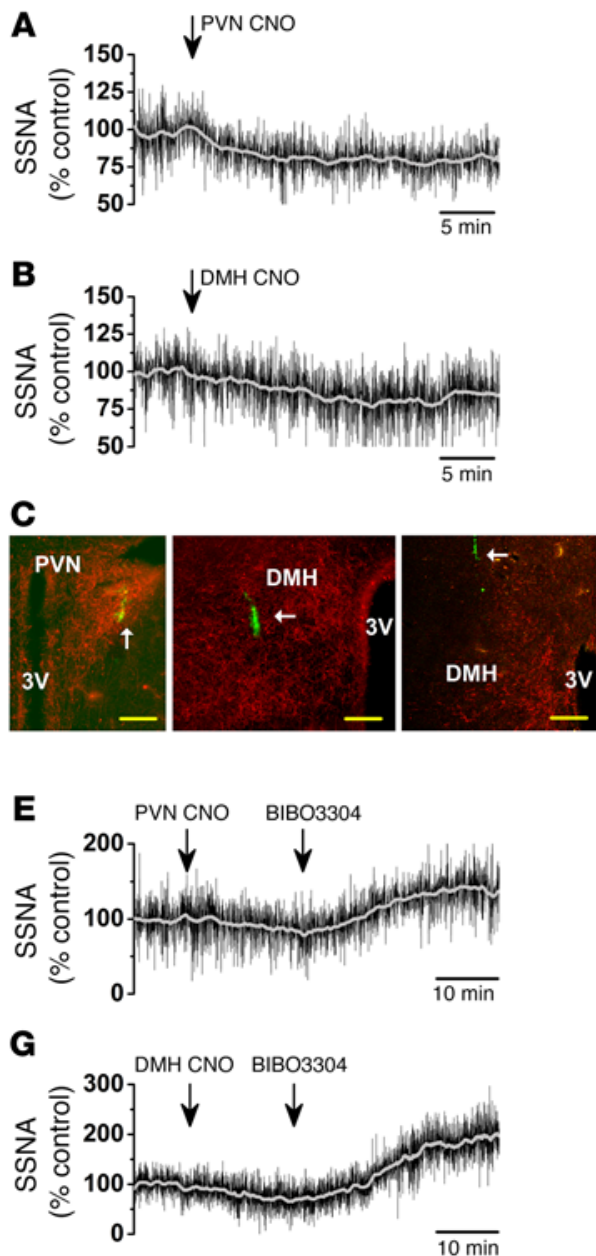
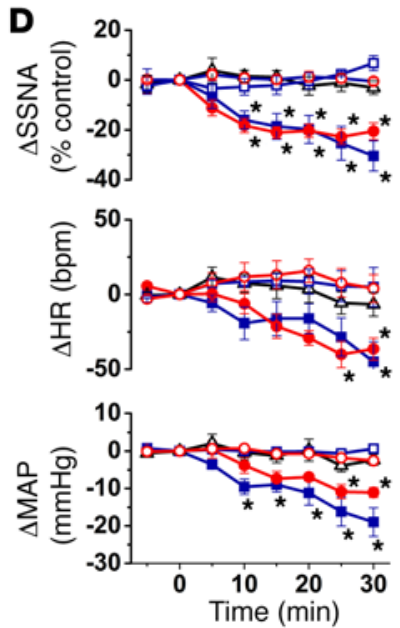

- PVN CNO $(n=7)$

- DMH CNO $(n=5)$

$\triangle$ Missed $(n=5)$

口PVN aCSF $(n=4)$

O DMH aCSF $(n=4)$

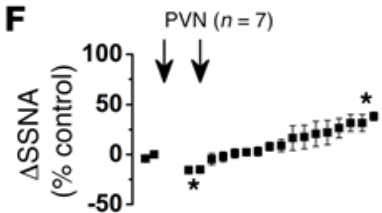

H

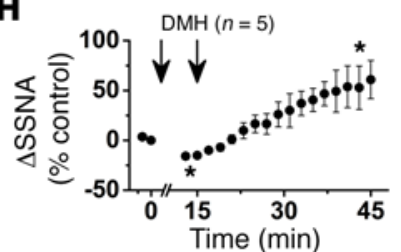

I

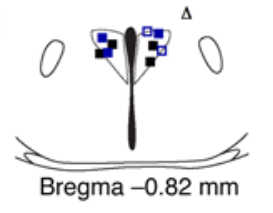

口PVN CNO
- PVN aCSF
DMH CNO

ODMH aCSF

$\triangle$ Missed

$\triangle$ Missed

and BIBO3304

0 O $\begin{aligned} & \mathrm{DMH} \mathrm{CNO}^{2} \\ & \text { and } \mathrm{BIBO} 3304\end{aligned}$
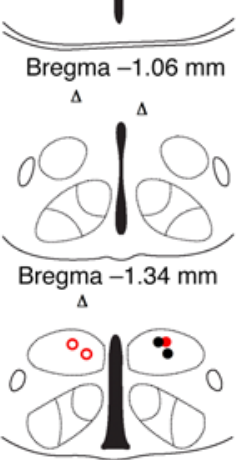

Bregma $-1.70 \mathrm{~mm}$
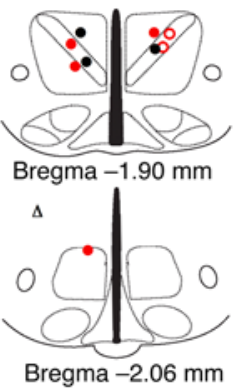

Figure 5. Bilateral nanoinjection of CNO into the PVN or DMH of ArcN hM3Dq mice decreases SSNA, HR, and MAP, and these responses are reversed by subsequent PVN or DMH injections of BIB03304. (A) Representative experiment showing that PVN CNO decreases SSNA in an ArcN hM3Dq mouse. (B) Representative experiment showing that DMH CNO decreases SSNA in an ArcN hM3Dq mouse. (C) Histological sections illustrating hM3Dq mCherrylabeled fibers from ArcN NPY/AgRP neurons and fluorescent injected beads in the PVN (left) and DMH (middle). The right panel shows an injection that missed the DMH. White arrows point to injection sites. Scale bars: $200 \mu \mathrm{m}$. (D) Group data showing that PVN or DMH CNO similarly decreases SSNA, HR, and MAP, but CNO injections that miss these targets or aCSF injections do not. Red symbols, DMH injections; blue symbols, PVN injections; black triangles, missed injections. Analyzed using 2-way repeated-measures ANOVA. (E) Representative experiment showing that PVN CNO decreases SSNA in a mouse harboring h3MDq in NPY/AgRP fibers, and this is reversed by PVN BIB03304. (F) Grouped data showing that PVN BIB03304 reverses the effects of PVN CNO. (G) Representative experiment showing that DMH CNO decreases SSNA in a mouse harboring h3MDq in NPY/AgRP fibers, and this is reversed by DMH BIB03304. (H) Grouped data showing that DMH BIBO3304 reverses the effects of DMH CNO. In F and $\mathbf{H}$, arrows indicate the times at which CNO, and then BIBO3304, were injected. Data in $\mathbf{F}$ and $\mathbf{H}$ were analyzed using 1-way repeated-measures ANOVA. Baseline values were $82 \pm 3 \mathrm{mmHg}$ and $492 \pm$ $13 \mathrm{bpm}$ (single PVN or DMH nanoinjections; $n=25$ ), $81 \pm 3 \mathrm{mmHg}$ and $461 \pm 21 \mathrm{bpm}$ (PVN CNO, followed by PVN BIBO3304; $n=7$ ), and $85 \pm 3 \mathrm{mmHg}$ and $452 \pm 24$ bpm (DMH CNO followed by DMH BIB03304; $n=5$ ). (I) Histological maps illustrating PVN and DMH injection sites (based on ref. 80$)$. ${ }^{*}<0.05$, compared with time 0 .

the substantial GABAergic tone already present. However, PVN GABA may contribute to the decreases in MAP.

DREADDs can be expressed in the terminal fields of targeted hypothalamic nuclei (15); therefore, we next tested whether local nanoinjection of CNO into the PVN (or DMH) decreases SSNA in ArcN hM3Dq mice. We chose a dose of CNO (30 nl of $10 \mu \mathrm{M} / \mathrm{l}$ ) that, when injected into the PVN of mice coexpressing ChR2 and hM4Di in ArcN AgRP neurons, maximally inhibited optogenetically evoked feeding (15). We found that PVN CNO (30 nl) promptly decreased SSNA, MAP, and HR, and interestingly these decreases were similar to those following nanoinjection of the same dose of
CNO into the DMH (Figure 5). Importantly, injection into sites that missed the PVN (or DMH) and injections of aCSF were ineffective (Figure 5). Moreover, in contrast to the inability of PVN BIBO3304 to reverse the sympathoinhibition evoked by i.p. CNO (Figure 4, $\mathrm{B}$ and $\mathrm{C}$ ), local BIBO3304 fully reversed the effects of CNO injections into the PVN and DMH (Figure 5, E-H), with peak SSNA increases (PVN: $32 \% \pm 6 \%$; DMH 55\% $\pm 13 \%$ ) similar to those following PVN BIBO3304 in WT mice (Figure 3, C and G) or in ArcN hM3Dq mice that received i.p. saline instead of CNO (Figure 4, C and G). Therefore, we conclude that ArcN NPY/AgRP neurons can also suppress SSNA via an action in the PVN, as well as in the DMH. 
A
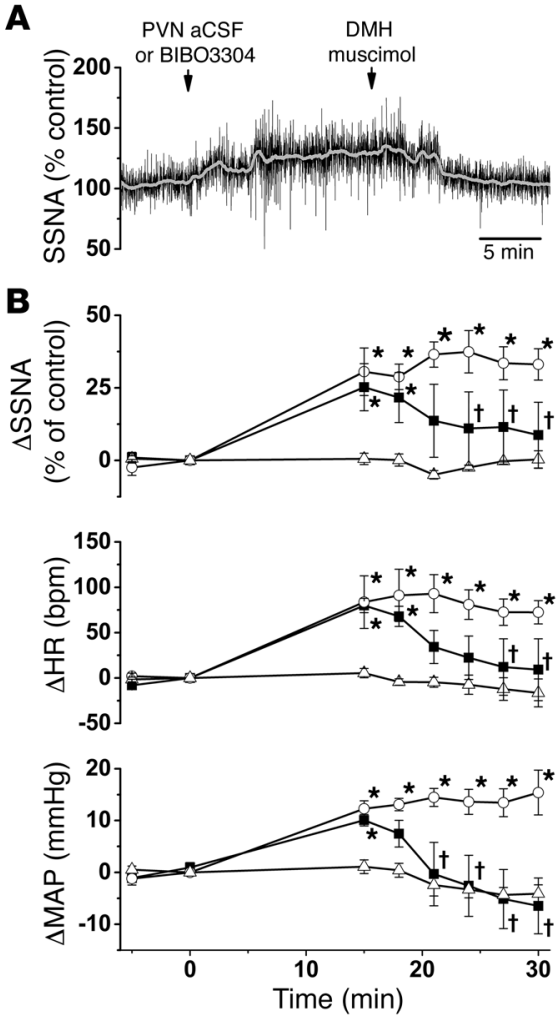

- PVN BIBO3304 + DMH muscimol $(n=4)$ OPVN BIBO3304 + missed DMH muscimol $(n=4)$ $\triangle \mathrm{PVN}$ aCSF $+\mathrm{DMH}$ muscimol $(n=5)$
C

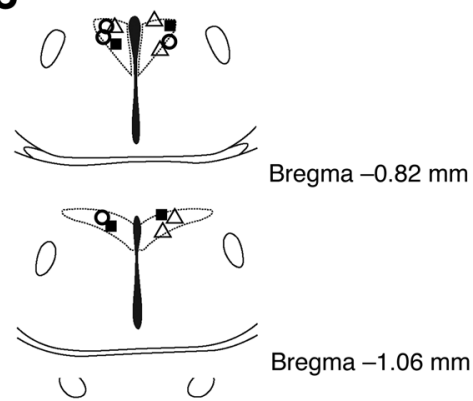

○

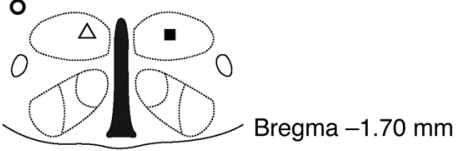

U
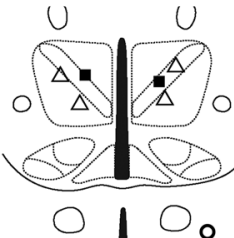

Bregma $-1.94 \mathrm{~mm}$
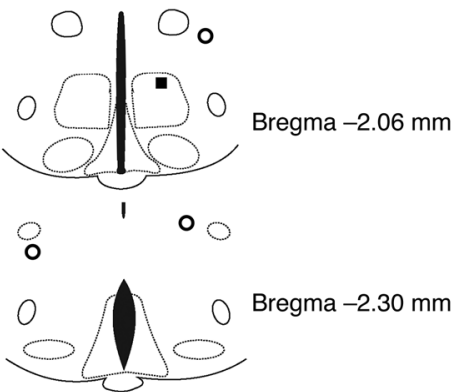

Figure 6. Inhibition of the DMH reverses the increases in SSNA, HR, and MAP induced by bilateral nanoinjections of BIBO3304 into the PVN. (A) Representative experiment showing that bilateral nanoinjections of muscimol into the $\mathrm{DMH}$ reverse the sympathoexcitatory effects of PVN BIB03304. (B) Grouped data showing that PVN BIB03304 increases SSNA, HR, and MAP, and bilateral DMH muscimol injections reverse these effects $(n=4)$. However, muscimol injections outside the DMH do not. (C) Histological maps illustrating PVN and DMH injection site (based on ref. 80). Baseline values were $83 \pm 3 \mathrm{mmHg}$ and $499 \pm 16 \mathrm{bpm}(n=13) .{ }^{*} P<0.05$, compared with time $0 ;{ }^{\dagger} P<0.05$ compared with value just before injection of muscimol.
Why, then, were PVN BIBO3304 injections after i.p. CNO not as effective as DMH BIBO3304 in reversing the sympathoinhibition elicited by hM3Dq-evoked plus endogenous tonic release of NPY? We hypothesized that in the PVN, the excitatory drive that increases SSNA after disinhibition with $\mathrm{BIBO} 3304$ is also inhibited by ArcN NPY/AgRP neurons. In support of this hypothesis, our anatomical studies revealed that ArcN NPY/AgRP neuronal fibers innervate regions that project to the PVN (34) and are also known to influence SNA: DMH, POA, PAG, and LPB (Figure 2 and Supplemental Figure 3). In support of such a role for the DMH, we found that the increase in SNA following PVN BIBO3304 was substantially reversed by DMH muscimol (Figure 6).

\section{Discussion}

It is well established that activation of ArcN NPY/AgRP neurons can trigger positive energy balance, largely by stimulating food intake, but also by reducing energy expenditure $(6,14,15,35$, $36)$. In contrast, the role of this neuronal population in the control of SNA had been largely unexplored, despite recent evidence that NPY acts in the PVN to inhibit SNA $(4,5,26)$ and that NPY/ AgRP neurons project to the PVN $(9,16,19)$. Here, via DREADD methodology, we demonstrate that ArcN AgRP/NPY neurons tonically suppress SNA, MAP, and HR. Moreover, using several approaches, we identified major projection targets - the PVN and $\mathrm{DMH}$ - through which ArcN NPY/AgRP neurons decrease SSNA. First, we demonstrated that presympathetic neurons that project to the RVLM are housed in the PVN, DMH, and LH of the mouse. RVLM-projecting neurons in both the PVN and DMH exhibited close associations with ArcN NPY/AgRP fibers, thereby providing an anatomical substrate for ArcN NPY-induced sympathoinhibition in these hypothalamic sites. Second, NPY decreased, and BIBO3304 increased, SSNA, MAP, and HR when injected into the PVN and DMH, but not the LH. We further demonstrated that the decreases in SSNA, MAP, and HR elicited by ArcN NPY/AgRP neuronal activation could be reversed by local blockade of DMH NPY1R. Finally, select activation of the terminal fields of ArcN NPY neurons in the PVN and DMH elicited prompt decreases in SSNA. Collectively, our studies identify ArcN NPY neurons as potent inhibitors of SNA, via projections to the PVN and DMH.

$\operatorname{ArcN}$ NPY neurons also release GABA and $\operatorname{AgRP}(37,38)$; therefore, chemogenetic activation of this population could potentially inhibit SNA via increased GABAergic or AgRP suppression of presympathetic neurons. However, nanoinjection of the GABA agonist muscimol into the PVN or DMH had minimal effects on SSNA or HR and only slightly decreased MAP. This result is not surprising given our finding in mice, as in rats (e.g. ref. 5), of pronounced tonic GABAergic inhibition of PVN and DMH presympathetic neurons, revealed by the dramatic increases in SSNA, MAP, and HR elicited by blockade of $\mathrm{GABA}_{\mathrm{A}}$ receptors in these regions. Thus, this high level of tonic inhibition would minimize the impact of further release of GABA. On the other hand, nanoinjections of NPY into the PVN (or DMH) are capable of eliciting 

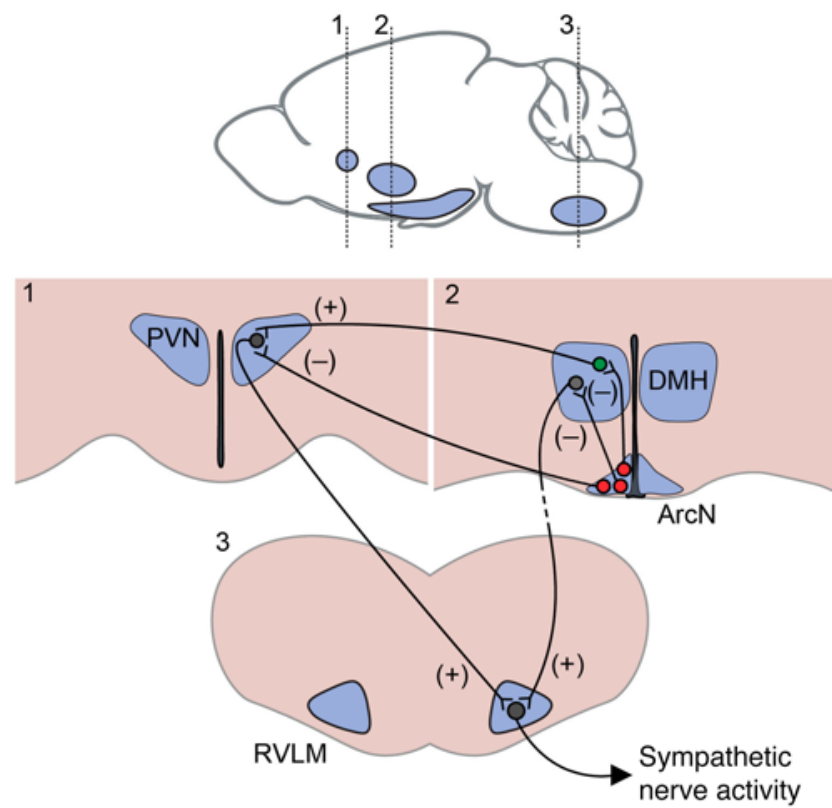

significant decreases in SNA, MAP, and HR, because NPY inhibits a $G$ protein-activated inwardly rectifying K (GIRK) current (39-41), which can hyperpolarize to a greater extent than $\mathrm{GABA}_{\mathrm{A}}$ receptors, which open $\mathrm{Cl}$ channels. While the limited inhibition by GABA was expected, the effect of PVN AgRP on SNA in rats is controversial $(8,42)$. Here, we show that doses of PVN AgRP that exceed those required to elicit food intake and sufficient to decrease body temperature failed to decrease SSNA, AP, or HR. Moreover, PVN BIBO3304 completely reversed the effects of CNO nanoinjections into the PVN, also suggesting that the effects of CNO-evoked release of GABA and AgRP are minimal. Interestingly, these results contrast sharply with the significant role documented for GABA and AgRP released from ArcN neurons in eliciting food intake $(24,43)$. Finally, it is noteworthy that blockade of DMH NPY1R completely reversed the effects of DREADD activation of ArcN NPY neurons, suggesting that NPY is the main mediator in this nucleus. Therefore, we conclude that the sympathoinhibition induced by stimulation of ArcN NPY/AgRP neurons is mediated largely by NPY. Nevertheless, decreases in the release of GABA could contribute to the increases in SSNA, MAP, or HR that occur following DREADD inhibition of ArcN NPY/AgRP neurons, although the rather large increases in SNA induced by blockade of NPY1R alone in the PVN or DMH suggest that tonic inhibition of PVN and DMH by NPY is nearly as efficacious as GABA, identifying ArcN NPY as a major inhibitory influence on SNA and AP.

Based on anatomical evidence that PVN presympathetic neurons are richly innervated by ArcN NPY fibers in mice (Figure 2) and rats (4), the strong sympathoinhibitory effects of NPY in the PVN in mice (Figure 3) and rats (4), and indirect evidence in rats that ArcN NPY neurons inhibit SNA via a synapse in the PVN (5, 7, 8), we were surprised that blockade of PVN NPY1R only weakly reversed the effects of select ArcN NPY/AgRP neuronal activation. In sharp contrast, blockade of DMH NPY1R completely reversed these effects. One explanation is that the ArcN-to-DMH pathway is more potent. However, the finding that the actions of PVN and DMH CNO equivalently decreased SSNA, MAP, and HR argues
Figure 7. Model illustrating proposed neurocircuitry by which ArcN NPY neurons inhibit SNA. Activation of ArcN NPY (red) neurons (e.g., by either systemic or local CNO administration) inhibits "presympathetic" neurons in both the PVN and DMH, and SNA decreases. The neuropathway by which NPY inputs into the DMH decrease SNA may be mediated in part by a direct projection to the RVLM, but also indirect projections (dashed line). On the other hand, our anatomical data (Figure 2) and previous studies in rats (4) suggest that ArcN-to-PVN NPY inputs directly inhibit presympathetic neurons that project to the RVLM (and possibly also the spinal cord). However, if so, then the failure of blockade of PVN NPY1R to reverse the effects of systemic CNO requires a mechanistic explanation. We hypothesize that reversal of CNO-induced NPY inhibition of PVN presympathetic neurons via PVN NPY1R blockade increases SNA; however, since DMH (and potentially other) neurons that drive the increase in SNA (green neuron) are still inhibited by the CNO-evoked activation of NPY1R, SNA does not reach a maximum. In contrast, we hypothesize that blockade of NPY1R in the DMH unmasks an unfettered excitatory input to RVLM neurons, resulting in a large increase in SNA.

against this possibility. Alternatively, the excitatory inputs into PVN that drive increased SNA following PVN BIBO3304 treatment are also inhibited by ArcN NPY/AgRP neurons. In other words, hM3Dq activation of ArcN NPY/AgRP neurons inhibits both PVN neurons and excitatory inputs to PVN neurons. After release of this inhibition via PVN BIBO3304 nanoinjections, an excitatory input into PVN that normally contributes to the increases in SNA remains inhibited, and SNA cannot increase above baseline. In support of this hypothesis, we found that AgRP/NPY fibers innervate the DMH, which projects heavily to the PVN (44-47). Moreover, nonselective inhibition of the DMH after PVN BIBO3304 treatment returned the elevations in SSNA, MAP, and HR toward baseline. Thus, these data identify the DMH as one source of excitatory drive following disinhibition of PVN presympathetic neurons. On the other hand, when NPY inhibition of DMH presympathetic neurons was reversed with BIBO3304, the excitatory input that increased SNA (perhaps from the PAG; ref. 48) appeared to remain undisturbed, and SNA rose above baseline. Additionally, the DMH receives a sizable brainstem NPY (catecholaminergic) projection $(49,50)$, which could contribute to basal NPY tone and be disinhibited by DMH BIBO3304. This is in contrast to brainstem NPY inputs to the PVN, which may be rare in the mouse (51).

To our knowledge, these data are the first in any species to identify an ArcN-to-DMH NPY projection that is capable of inhibiting cardiovascularly relevant SNA. Consequently, the downstream neurocircuitry by which NPY inputs to the DMH are able to influence SNA and AP is unknown. Previous studies have documented that the DMH is an important node in sympathoexcitatory responses, in particular following psychological stress (for reviews, see refs. $48,52,53)$. A key component of this stress-induced descending pathway includes the RVLM $(33,54,55)$. However, retrograde tracer studies in rats largely conclude that DMH neurons projecting directly to the RVLM are uncommon (44, 45 , 56). In the mouse DMH, we observed a significant number of RVLM-projecting neurons; however, the number was far smaller than when the CtB injections encompassed the raphe pallidus 
(Supplemental Figure 10), a well-described projection target in both rats and mice $(11,47)$. Moreover, only a modest number of DMH neurons that were retrogradely labeled from the RVLM were closely associated with ArcN NPY neurons traced with a synaptophysin mCherry label (Figure 2), although we cannot discount the possible inhibitory influence of NPY on DMH-to-RVLM neurons via volume transmission $(20,21)$. Thus, while NPY may influence a DMH-to-RVLM cohort, a relay intermediate between the DMH and RVLM may also be involved. Prior neuroanatomical studies in the rat (44-46) and mouse (47) reveal that the DMH heavily innervates many preautonomic regions, particularly in the forebrain. A very dense projection is to the caudal parvocellular PVN, a hypothalamic subnucleus that projects to the RVLM and the spinal cord (44-47). The median POA, the perifornical area, and the PAG are also major projection targets $(45,47)$. Future studies are required to specifically delineate the neuropathways involved.

The present results reveal that ArcN NPY neurons tonically suppress SNA and AP, likely in part via a projection to PVN neurons that target the RVLM, the source of tonic sympathetic tone (Figure 7). We also identify a parallel sympathoinhibitory ArcN NPY input to the $\mathrm{DMH}$, although the downstream neurocircuitry remains to be determined (Figure 7). What is the physiological significance of these findings? ArcN NPY/AgRP neuronal activity and PVN NPY concentrations increase to reach a peak just before an anticipated meal and remain elevated for a period after eating commences (57-60). Ghrelin, which is released from the stomach when it is emptied, also peaks right before eating and likely contributes to the increased hypothalamic NPY content (61). In general, ArcN NPY actions are anticipatory, driving increased energy stores. NPY increases appetite (6) and mastication (62), suppresses thermogenesis in brown adipose tissue (62), and, like ghrelin (61), increases lipogenesis in both white adipose tissue (WAT) and the liver (for reviews, see refs. 63, 64). The sympathetic nervous system likely contributes to a subset of these actions. For example, an NPY-induced decrease in SSNA to vascular smooth muscle would favor increased blood flow to the gastrointestinal tract and liver, to enhance nutrient absorption and storage. Indeed, we found that the majority of SSNA is inhibited by baroreceptor activation via an increase in AP (Supplemental Figure 11), suggesting that much of SSNA is related to cardiovascular function (i.e., innervating blood vessels). Nevertheless, as insulin rises in the postabsorptive state, it inhibits NPY (7) and acts centrally to increase SSNA (29), in part to counter its own direct vasodilatory action. NPY-induced decreases in SSNA may also influence metabolism. For example, the ability of NPY to promote hepatic lipogenesis depends on the sympathetic nervous system (i.e., NPY-evoked lipogenesis is eliminated by hepatic denervation); however, whether this effect is mediated by an increase or decrease in SNA has not been directly determined. The present results suggest that this action, to promote hepatic lipogenesis, may be due to a decrease in SNA. Finally, we also speculate that the central action of NPY (or decreased melanocortins) to increase WAT lipogenesis $(64,65)$ is also mediated by NPY-induced decreases in SNA. The literature provides support: the melanocortin agonist MTII increases WAT SNA (65), and a decrease in WAT SNA inhibits lipolysis (66).

These results may also have pathophysiological implications. Obesity and leptin increase SNA, which can lead to hypertension (for reviews, see refs. 1-3, 67). Moreover, obesity can decrease ArcN or PVN NPY content or mRNA levels, particularly with time after initiation of a high-fat diet $(6,68-72)$. There are few studies of the DMH, but recent evidence suggests that a high-fat diet or leptin decreases DMH NPY1R expression in males, but interestingly not in females $(73,74)$. Therefore, because a decrease in NPY tone is required for POMC neurons to induce sympathoexcitation, at least in the PVN $(4,5)$, reduced tonic NPY sympathoinhibitory actions (due to decreased NPY release or NPY1R) in both PVN and $\mathrm{DMH}$ may represent a fruitful target for understanding the mechanistic underpinnings of obesity-induced sympathoexcitation. Moreover, given the interest in NPY agonists and antagonists as therapeutic agents (13), our data may also inform their use in the treatment of such sympathoexcitatory disorders.

\section{Methods}

\section{Animals}

Male mice (20-30 g) were used. Colonies of mice were bred in-house. C57BL/6J mice (WT) were obtained from the Jackson Laboratory. Agrp-IRES-Cre mice (75) were originally obtained from the Jackson Laboratory. NPY-hrGFP transgenic mice were originally received from Bradford B. Lowell (Harvard University, Boston, Massachusetts, USA) (76). Heterozygous Agrp-IRES-Cre NPY-hrGFP (Agrp $\left.{ }^{C r e}:: N p y^{G F P}\right)$ mice were produced in-house by breeding of the respective strains.

Mice were housed under constant temperature $\left(21-23^{\circ} \mathrm{C}\right)$ and a 12-hour light/12-hour dark cycle schedule (lights on at 6 am and lights off at $6 \mathrm{pm}$ ), with food (LabDiet 5LOD) and water provided ad libitum.

\section{Brain viral vector and $\mathrm{CtB}$ injections}

Stereotaxic AAV-DREADD-mCherry injections. Male Agrp-IRES-Cre mice, WT littermates, and $A g R P^{C r e}:: N p y^{G F P}$ mice received bilateral ArcN injections of AAV8-hSyn-DIO-hM3Dq-mCherry or AAV8hSyn-DIO-hM4Di-mCherry (UNC Vector Core Services, Chapel Hill, North Carolina, USA). The mice were anesthetized with isoflurane (1.5\%-2.5\%), placed in a stereotaxic instrument (Model 1900, David Kopf Instruments) and received a midline skin incision to expose the surface of the skull. Two sets of $250 \mathrm{nl}$ bilateral injections were made in the ArcN (coordinates: 1.0 and $1.6 \mathrm{~mm}$ caudal to bregma, $0.3 \mathrm{~mm}$ lateral to midline, $5.8 \mathrm{~mm}$ ventral to dura). The injections were conducted bilaterally over 1 minute with a glass pipette (tip diameter, 40 $\mu \mathrm{m})$ connected to a Nanoject II (Drummond Scientific). The injector was left in place for 10 minutes before withdrawal. Mice were allowed to recover for at least 2 weeks before the start of any in vivo studies. At the conclusion of the study, the brains were fixed with $4 \%$ paraformaldehyde, then sectioned at $30 \mu \mathrm{m}$ on a vibrating microtome, and processed for immunohistochemical detection of mCherry. All injected Agrp-IRES-Cre mice were mCherry positive for bilateral hits.

Stereotaxic injection for tract tracing. $250 \mathrm{nl}$ of AAV8.2-hEF1alpha-DIO-Synaptophysin-mCherry-WPRE (77, 78) (VIROVEK) were injected into the $\mathrm{ArcN}$ as described above. One week after the surgery, the mice received another stereotaxic injection of Alexa Fluor $488^{-}$CtB $(1 \mathrm{mg} / \mathrm{ml}, 30 \mathrm{nl}$, Life Technologies, Invitrogen) into the RVLM (coordinates: 1.5 to $1.6 \mathrm{~mm}$ caudal to lambda, 0.9 to 1.1 $\mathrm{mm}$ lateral to midline, 4.9 to $5.1 \mathrm{~mm}$ ventral to dura) using a pressure injection system. One week later, the animals were perfused with $4 \%$ paraformaldehyde, and the brains were sectioned at $30 \mu \mathrm{m}$ on a 
freezing-stage microtome and processed for immunohistochemical detection of mCherry and CtB.

\section{Experimental preparation: AP and SNA recordings}

Surgery. Mice were anesthetized with 1.5\%-2.5\% isoflurane in $100 \%$ oxygen, and intubated to allow for spontaneous respiration of oxygen-enriched room air. Rectal temperature was maintained at $36-38^{\circ} \mathrm{C}$ using a rectal thermistor and heating pad. The splanchnic nerve was identified using a dissecting scope, mounted on a bipolar stainless steel electrode, and then embedded with silicone gel (Kwik-Sil, World Precision Instruments). The right jugular vein was cannulated to maintain anesthesia after surgery with $\alpha$-chloralose (initial dose: $25 \mathrm{mg} / \mathrm{kg}$; sustaining dose of $6 \mathrm{mg} / \mathrm{kg} / \mathrm{h}$; Sigma-Aldrich) (79). The left carotid artery was cannulated for continuous measurement of AP and HR. The mice were then placed in a Kopf stereotaxic instrument, and, following a midline incision, the skull was prepared for nanoinjections by burring a hole in the skull near the midline. For i.p. injections, a PE-10 tube filled with saline was implanted into the peritoneal cavity in some mice. After completion of surgery and the $\alpha$-chloralose loading dose, the animals were allowed to stabilize for $\geq 30$ minutes before experimentation. After experimentation, all mice were killed with an overdose of pentobarbital. Postmortem nerve activity was recorded in all animals.

Data acquisition. Pulsatile and mean AP, HR, and raw SSNA were continuously recorded throughout the experiment with Grass amplifiers (model 79D, Grass Instrument Co.) and a Biopac MP100 data acquisition and analysis system (BIOPAC Systems Inc.), sampling at 2,000 Hz. SNA was bandpass filtered $(20-3,000 \mathrm{~Hz})$ and amplified $(\times 10,000)$. The SSNA signal was then rectified and integrated in 1 -second bins. After data collection, background postmortem SSNA was subtracted from values of SSNA recorded during the experiment. SSNA was normalized to the baseline (or control) SNA, which was defined as the average of the 60 -second period before the first injections (i.p. injection or nanoinjection) and expressed as percentage of control. Baseline SSNA, MAP, and HR were 1-minute averages prior to injections, and response values were the difference of the averages of 1-minute bins following injections from the baseline value.

\section{Hypothalamic nanoinjections}

Nanoinjections were conducted with single-barreled glass micropipettes with $\sim 20-\mu \mathrm{m}$ tip diameter. With flat-skull positioning and using bregma as zero, micropipettes were positioned using the following coordinates: PVN, 0.7-0.9 mm caudal, 0.3-0.4 mm lateral, and $4.8 \mathrm{~mm}$ ventral; $\mathrm{DMH}$, 1.7-1.9 mm caudal, $0.4 \mathrm{~mm}$ lateral, and $5.1 \mathrm{~mm}$ ventral; and LH, 1.3-1.5 $\mathrm{mm}$ caudal, $1.0 \mathrm{~mm}$ lateral, and 5.0-5.1 $\mathrm{mm}$ ventral. All nanoinjections (30 nl/side) were made bilaterally with $\sim 2$ minutes between injections, using a pressure injection system (Pressure System IIe, Toohey Co.).

The following drugs and chemicals were used for nanoinjections: NPY, 0.1 mM/l (Tocris Bioscience); BIBO3304, NPY1R antagonist, 10 mM/l (Tocris Bioscience); AgRP, 1 mM/l (Phoenix Pharmaceuticals Inc.); muscimol, $1 \mathrm{mM} / 1$ (Tocris Bioscience); CNO, $10 \mu \mathrm{M} / 1$ (Enzo Life Sciences); and gabazine, $0.03 \mathrm{mM} / 1$ and $0.1 \mathrm{mM} / \mathrm{l}$ (SR-95531, Sigma-Aldrich). Drugs for microinjection were dissolved in aCSF containing (in mM): $128 \mathrm{NaCl}, 2.6 \mathrm{KCl}, 1.3 \mathrm{CaCl}_{2}, 0.9 \mathrm{MgCl}_{2}, 20 \mathrm{NaHCO}_{3}$, $1.3 \mathrm{Na}_{2} \mathrm{HPO}_{4}$, and 2 dextrose; $\mathrm{pH}$ 7.4. In some animals, prior to nanoinjections of drugs, aCSF was injected as vehicle control. Fluorescent polystyrene microspheres (FluoSpheres, F8803, 1:200; Molecular Probes) were included in the injectate to verify the injection sites.
At the conclusion of the experiment, the brains were removed and placed in $4 \%$ paraformaldehyde for at least 48 hours. The hypothalamus was subsequently cut into $50-\mu \mathrm{m}$ sections using a vibrating microtome and counterstained with Hoechst 34580 (1:500, SigmaAldrich). Correct placement was confirmed using a mouse brain atlas (80) under a Zeiss Axiophot 2 Plus fluorescence microscope (Carl Zeiss Microscopy).

\section{Experimental protocols}

(i) hM3Dq or hM4Di activation: After stabilization, saline or CNO ( 0.3 or $1 \mathrm{mg} / \mathrm{kg}$, i.p., in $0.1 \mathrm{ml}$ ) was injected in Agrp-IRES-Cre mice or their WT littermates that had received nanoinjections of hM3Dq or hM4Di AAV into the ArcN; and AP, HR, and SSNA were recorded for more than 1 hour following injections.

(ii) Single hypothalamic drug nanoinjections: In male WT mice, after collection of baseline data, nanoinjections (30 nl, over 5-10 seconds) were made bilaterally, with $\sim 2$ minutes between injections, and data were collected for the following 30 minutes.

(iii) Reversal of hM3Dq activation-induced effects via BIBO3304 nanoinjections in the PVN or DMH: In Agrp-IRES-Cre mice with hM3Dq, 35-40 minutes after i.p. injection of saline or CNO, BIBO3304 $(10 \mathrm{mM} / \mathrm{l})$ was injected bilaterally into the PVN or into the $\mathrm{DMH}$, and data were collected for 30 minutes. In another set of mice, i.p. CNO was followed by bilateral PVN or DMH injections of aCSF.

(iv) Two hypothalamic drug nanoinjections: In male WT mice, two different drugs were injected bilaterally (as described above) at an interval of $\sim 15$ minutes, when the effects of the first injection became stable. The combinations of nanoinjections include: NPY in the PVN, followed by BIBO3304 in the PVN; BIBO3304 in the PVN, followed by muscimol or aCSF into the DMH. In addition, in male Agrp-IRESCre mice that received ArcN hM3Dq, two different drugs were injected bilaterally (as described above) at an interval of $\sim 15$ minutes: CNO in the PVN followed by BIBO3304 in the PVN; CNO in the DMH followed by BIBO3304 in the DMH.

\section{Food intake studies}

Food intake measurements were performed as previously described (14). Mice were singly housed for at least 1 day before the experiment. AgrpIRES-Cre mice with hM3Dq randomly received i.p. injections of CNO ( $0.3 \mathrm{mg} / \mathrm{kg}$ of body weight, i.p.) or saline 3 hours after the start of the 12-hour light cycle ( $9 \mathrm{am}$ ) and the other solution 2 days later. Thus, each animal was used as its own control. Food intake was assessed by providing one pellet of food in a petri dish in the cage, and weighing the pellet before and at 2 and 4 hours after the injection. Agrp-IRES-Cre mice with hM4Di were randomly injected with CNO $(0.3 \mathrm{mg} / \mathrm{kg}$ of body weight, i.p.) or saline at the start of the 12-hour dark cycle $(6 \mathrm{pm})$ on alternating days, and food intake was assessed at 2 and 4 hours after injection.

\section{Immunohistochemistry}

Immunohistochemistry was performed as previously described $(4,26$, $81)$. The primary antibodies used in these studies included rabbit antidsRed, which detects mCherry (1:2,500; catalog 632496, Clontech), rabbit anti-mCherry (1:1,000; ab167453, Abcam), and goat anti-CtB (1:10,000; product 703, List Biological Laboratories). mCherry and $\mathrm{CtB}$ were then visualized with the secondary antibodies Alexa Fluor 594-donkey anti-rabbit (1:500; catalog A21207, Invitrogen) and Alexa Fluor 488-donkey anti-goat (1:500; catalog A11055, Invitrogen). 
Images were collected using a Zeiss LSM 780 laser scanning confocal microscope (Carl Zeiss MicroImaging Inc.). The number of ArcN hM3Dq-expressing neurons also positive for NPY GFP (Supplemental Figure $1 \mathrm{E}$ ) was analyzed offline using the image processing software Fiji (4). RVLM-projecting neurons in the PVN, DMH, and LH closely associated with ArcN NPY/AgRP fibers or terminals were quantified as follows (Supplemental Figure 12): images were processed in Zeiss Zen 2012 (Gray version) (Carl Zeiss Microimaging Inc.) and imported into Imaris 8.4.1 (Bitplane, Andor Technologies). The two-channel (red and green) thresholds were adjusted to minimize background, and CtB-labeled cells (green) were given an isosurface. Spots representing mCherry-labeled fibers and terminals (red) were created in the same manner and were divided using distance transformation into those $2 \mu \mathrm{m}$ or closer to a green cell surface and those further away. Identical parameters were applied to each image. The number of green cells having red contacts was determined.

\section{Statistics}

All data are presented as mean \pm SEM. Between-group differences were determined using 2-way repeated-measures ANOVA, except for data displayed in Figure 5 and Supplemental Figure 6, for which 1-way ANOVA was used. Specific between- and within-group differences were determined using the Newman-Keuls post hoc test. $P<0.05$ was considered statistically significant.

\section{Study approval}

All procedures were conducted in accordance with the NIH's Guide for the Care and Use of Laboratory Animals (National Academies Press,
2011) and were approved by the Oregon Health \& Science University Institutional Animal Care and Use Committee.

\section{Author contributions}

ZS performed all in vivo experiments and viral vector injections. ZS and CJM surgically prepared mice for immunocytochemical analysis. ZS, CJM, and VLB designed the experiments, analyzed the data, and prepared the manuscript.

\section{Acknowledgments}

We gratefully acknowledge the technical support provided by Cole T. Streiff and Rosalee Elting (genotyping), Katelyn Castellanos and Alexia Robles (histological sectioning), Rubing Xing (immunohistochemistry), Nicole Pelletier, and Stefanie Kaech Petrie and Aurelie Snyder (confocal imaging and quantification). We also thank Donald Morgan (University of Iowa, Kamal Rahmouni laboratory) for advice as we developed the anesthetized mouse preparation. Finally, we appreciate the comments made by Martin Kelly and Daniel Marks during preparation of the manuscript. This research was funded in part by NIH grants HL088552 and HL128181 (VLB), AHA15POST23040042 postdoctoral fellowship (ZS), ADA 1-13-BS-120 (CJM), and NINDS P30 NS061800 (PI, S. Aicher).

Address correspondence to: Virginia L. Brooks, Oregon Health \& Science University, Department of Physiology and Pharmacology, 1381 SW Sam Jackson Park Road - L334, Portland, Oregon 97239, USA. Phone: 503.494.5843; Email: brooksv@ohsu.edu.
1. Davy KP, Orr JS. Sympathetic nervous system behavior in human obesity. Neurosci Biobehav Rev. 2009;33(2):116-124.

2. Grassi G, Mark A, Esler M. The sympathetic nervous system alterations in human hypertension. Circ Res. 2015;116(6):976-990.

3. Hall JE, et al. Obesity-induced hypertension: role of sympathetic nervous system, leptin, and melanocortins. J Biol Chem. 2010;285(23):17271-17276.

4. Cassaglia PA, et al. Neuropeptide Y acts in the paraventricular nucleus to suppress sympathetic nerve activity and its baroreflex regulation. J Physiol (Lond). 2014;592(7):1655-1675.

5. Shi Z, Li B, Brooks VL. Role of the paraventricular nucleus of the hypothalamus in the sympathoexcitatory effects of leptin. Hypertension. 2015;66(5):1034-1041.

6. Beck B. Neuropeptide Y in normal eating and in genetic and dietary-induced obesity. Philos Trans R Soc Lond, B, Biol Sci. 2006;361(1471):1159-1185.

7. Cassaglia PA, Shi Z, Brooks VL. Insulin increases sympathetic nerve activity in part by suppression of tonic inhibitory neuropeptide $\mathrm{Y}$ inputs into the paraventricular nucleus in female rats. Am J Physiol Regul Integr Comp Physiol. 2016;311(1):R97-R103.

8. Kawabe T, Kawabe K, Sapru HN. Cardiovascular responses to chemical stimulation of the hypothalamic arcuate nucleus in the rat: role of the hypothalamic paraventricular nucleus. PLOS ONE. 2012;7(9):e45180.

9. Grove KL, Smith MS. Ontogeny of the hypotha- lamic neuropeptide Y system. Physiol Behav. 2003;79(1):47-63.

10. Sawchenko PE, Swanson LW, Grzanna R, Howe PR, Bloom SR, Polak JM. Colocalization of neuropeptide Y immunoreactivity in brainstem catecholaminergic neurons that project to the paraventricular nucleus of the hypothalamus. J Comp Neurol. 1985;241(2):138-153.

11. Lee SJ, et al. Efferent projections of neuropeptide Y-expressing neurons of the dorsomedial hypothalamus in chronic hyperphagic models. J Comp Neurol. 2013;521(8):1891-1914.

12. Reichmann F, Holzer P. Neuropeptide Y: a stressful review. Neuropeptides. 2016;55:99-109.

13. Brothers SP, Wahlestedt C. Therapeutic potential of neuropeptide Y (NPY) receptor ligands. EMBO MolMed. 2010;2(11):429-439.

14. Krashes MJ, et al. Rapid, reversible activation of AgRP neurons drives feeding behavior in mice. J Clin Invest. 2011;121(4):1424-1428.

15. Stachniak TJ, Ghosh A, Sternson SM. Chemogenetic synaptic silencing of neural circuits localizes a hypothalamus $\rightarrow$ midbrain pathway for feeding behavior. Neuron. 2014;82(4):797-808.

16. Broberger C, Johansen J, Johansson C, Schalling M, Hökfelt T. The neuropeptide Y/agouti gene-related protein (AGRP) brain circuitry in normal, anorectic, and monosodium glutamate-treated mice. Proc Natl Acad Sci U S A. 1998;95(25):15043-15048.

17. MacLaren DA, et al. Clozapine N-oxide administration produces behavioral effects in Long-
Evans rats: Implications for designing DREADD experiments. eNeuro. 2016;3(5): ENEURO. 0219-16.2016.

18. Jann MW, Lam YW, Chang WH. Rapid formation of clozapine in guinea-pigs and man following clozapine-N-oxide administration. Arch Int Pharmacodyn Ther. 1994;328(2):243-250.

19. Bagnol D, et al. Anatomy of an endogenous antagonist: relationship between Agouti-related protein and proopiomelanocortin in brain. $\mathrm{JNeu}$ rosci. 1999;19(18):RC26.

20. Kopp J, et al. Expression of the neuropeptide $Y$ Y1 receptor in the CNS of rat and of wild-type and Y1 receptor knock-out mice. Focus on immunohistochemical localization. Neuroscience. 2002;111(3):443-532.

21. Sørensen AT, Kanter-Schlifke I, Lin EJ, During MJ, Kokaia M. Activity-dependent volume transmission by transgene NPY attenuates glutamate release and LTP in the subiculum. Mol Cell Neurosci. 2008;39(2):229-237.

22. Fetissov SO, Kopp J, Hökfelt T. Distribution of NPY receptors in the hypothalamus. Neuropeptides. 2004;38(4):175-188.

23. Fetissov SO, Byrne LC, Hassani H, Ernfors P, Hökfelt T. Characterization of neuropeptide Y Y2 and $\mathrm{Y} 5$ receptor expression in the mouse hypothalamus. JComp Neurol. 2004;470(3):256-265.

24. Atasoy D, Betley JN, Su HH, Sternson SM. Deconstruction of a neural circuit for hunger. Nature. 2012;488(7410):172-177.

25. Sutton AK, Pei H, Burnett KH, Myers MG, 
Rhodes CJ, Olson DP. Control of food intake and energy expenditure by Nos1 neurons of the paraventricular hypothalamus. J Neurosci. 2014;34(46):15306-15318.

26. Shi Z, Cassaglia PA, Gotthardt LC, Brooks VL. Hypothalamic paraventricular and arcuate nuclei contribute to elevated sympathetic nerve activity in pregnant rats: roles of neuropeptide $\mathrm{Y}$ and $\alpha$-melanocyte-stimulating hormone. Hypertension. 2015;66(6):1191-1198.

27. Joppa MA, Ling N, Chen C, Gogas KR, Foster AC, Markison S. Central administration of peptide and small molecule MC4 receptor antagonists induce hyperphagia in mice and attenuate cytokine-induced anorexia. Peptides. 2005;26(11):2294-2301.

28. Sutton GM, et al. The melanocortin-3 receptor is required for entrainment to meal intake. J Neurosci. 2008;28(48):12946-12955.

29. Cassaglia PA, Hermes SM, Aicher SA, Brooks VL. Insulin acts in the arcuate nucleus to increase lumbar sympathetic nerve activity and baroreflex function in rats. J Physiol (Lond). 2011;589(Pt 7):1643-1662.

30. Pina MM, Young EA, Ryabinin AE, Cunningham CL. The bed nucleus of the stria terminalis regulates ethanol-seeking behavior in mice. Neuropharmacology. 2015;99:627-638.

31. Flint RR, Chang T, Lydic R, Baghdoyan HA. GABA(A) receptors in the pontine reticular formation of C57BL/6J mouse modulate neurochemical, electrographic, and behavioral phenotypes of wakefulness. J Neurosci. 2010;30(37):12301-12309.

32. Madden CJ, Morrison SF. Neurons in the paraventricular nucleus of the hypothalamus inhibit sympathetic outflow to brown adipose tissue. Am J Physiol Regul Integr Comp Physiol. 2009;296(3):R831-R843.

33. Cao WH, Fan W, Morrison SF. Medullary pathways mediating specific sympathetic responses to activation of dorsomedial hypothalamus. Neuroscience. 2004;126(1):229-240.

34. Gautron L, Cravo RM, Elmquist JK, Elias CF. Discrete melanocortin-sensitive neuroanatomical pathway linking the ventral premmamillary nucleus to the paraventricular hypothalamus. Neuroscience. 2013;240:70-82.

35. Chambers AP, Woods SC. The role of neuropeptide $\mathrm{Y}$ in energy homeostasis. Handb Exp Pharmacol. 2012;(209):23-45.

36. Shi YC, et al. Arcuate NPY controls sympathetic output and BAT function via a relay of tyrosine hydroxylase neurons in the PVN. Cell Metab. 2013;17(2):236-248.

37. Wu Q, Palmiter RD. GABAergic signaling by AgRP neurons prevents anorexia via a melanocortin-independent mechanism. Eur J Pharmacol. 2011;660(1):21-27.

38. Sapru HN. Role of the hypothalamic arcuate nucleus in cardiovascular regulation. Auton Neurosci. 2013;175(1-2):38-50.

39. Roseberry AG, Liu H, Jackson AC, Cai X, Friedman JM. Neuropeptide Y-mediated inhibition of proopiomelanocortin neurons in the arcuate nucleus shows enhanced desensitization in ob/ob mice. Neuron. 2004;41(5):711-722.

40. Acuna-Goycolea C, Tamamaki N, Yanagawa Y, Obata K, van den Pol AN. Mechanisms of neuropeptide Y, peptide YY, and pancreatic polypeptide inhibition of identified green fluorescent protein-expressing GABA neurons in the hypothalamic neuroendocrine arcuate nucleus. J Neurosci. 2005;25(32):7406-7419.

41. Fu LY, Acuna-Goycolea C, van den Pol AN. Neuropeptide $\mathrm{Y}$ inhibits hypocretin/orexin neurons by multiple presynaptic and postsynaptic mechanisms: tonic depression of the hypothalamic arousal system. J Neurosci. 2004;24(40):8741-8751.

42. Li P, Cui BP, Zhang LL, Sun HJ, Liu TY, Zhu GQ. Melanocortin 3/4 receptors in paraventricular nucleus modulate sympathetic outflow and blood pressure. Exp Physiol. 2013;98(2):435-443.

43. Krashes MJ, Shah BP, Koda S, Lowell BB. Rapid versus delayed stimulation of feeding by the endogenously released AgRP neuron mediators GABA, NPY, and AgRP. Cell Metab. 2013;18(4):588-595.

44. Ter Horst GJ, Luiten PG. Phaseolus vulgaris leuco-agglutinin tracing of intrahypothalamic connections of the lateral, ventromedial, dorsomedial and paraventricular hypothalamic nuclei in the rat. Brain Res Bull. 1987;18(2):191-203.

45. Thompson RH, Canteras NS, Swanson LW. Organization of projections from the dorsomedial nucleus of the hypothalamus: a PHA-L study in the rat. JComp Neurol. 1996;376(1):143-173.

46. Dai J, Van Der Vliet J, Swaab DF, Buijs RM. Postmortem anterograde tracing of intrahypothalamic projections of the human dorsomedial nucleus of the hypothalamus. J Comp Neurol. 1998;401(1):16-33.

47. Zhang Y, et al. Leptin-receptor-expressing neurons in the dorsomedial hypothalamus and median preoptic area regulate sympathetic brown adipose tissue circuits. J Neurosci. 2011;31(5):1873-1884

48. Fontes MA, Xavier CH, de Menezes RC, Dimicco JA. The dorsomedial hypothalamus and the central pathways involved in the cardiovascular response to emotional stress. Neuroscience. 2011;184:64-74.

49. Stornetta RL, Akey PJ, Guyenet PG. Location and electrophysiological characterization of rostral medullary adrenergic neurons that contain neuropeptide Y mRNA in rat medulla. J Comp Neurol. 1999;415(4):482-500.

50. Abbott SB, DePuy SD, Nguyen T, Coates MB, Stornetta RL, Guyenet PG. Selective optogenetic activation of rostral ventrolateral medullary catecholaminergic neurons produces cardiorespiratory stimulation in conscious mice. J Neurosci. 2013;33(7):3164-3177.

51. Broberger C, Visser TJ, Kuhar MJ, Hökfelt T. Neuropeptide $Y$ innervation and neuropeptide-Y-Y1-receptor-expressing neurons in the paraventricular hypothalamic nucleus of the mouse. Neuroendocrinology. 1999;70(5):295-305.

52. DiMicco JA, Samuels BC, Zaretskaia MV, Zaretsky DV. The dorsomedial hypothalamus and the response to stress: part renaissance, part revolution. Pharmacol Biochem Behav. 2002;71(3):469-480.

53. Dampney RA. Central mechanisms regulating coordinated cardiovascular and respiratory function during stress and arousal. Am J Physiol Regul Integr Comp Physiol. 2015;309(5):R429-R443.
54. Fontes MA, Tagawa T, Polson JW, Cavanagh SJ, Dampney RA. Descending pathways mediating cardiovascular response from dorsomedial hypothalamic nucleus. Am J Physiol Heart Circ Physiol. 2001;280(6):H2891-H2901.

55. Horiuchi J, McAllen RM, Allen AM, Killinger S, Fontes MA, Dampney RA. Descending vasomotor pathways from the dorsomedial hypothalamic nucleus: role of medullary raphe and RVLM. Am J Physiol Regul Integr Comp Physiol. 2004;287(4):R824-R832.

56. Papp RS, Palkovits M. Brainstem projections of neurons located in various subdivisions of the dorsolateral hypothalamic area-an anterograde tract-tracing study. Front Neuroanat. 2014;8:34.

57. Yoshihara T, Honma S, Honma K. Effects of restricted daily feeding on neuropeptide $\mathrm{Y}$ release in the rat paraventricular nucleus. $A m J$ Physiol. 1996;270(4 Pt 1):E589-E595.

58. Kalra SP, Dube MG, Sahu A, Phelps CP, Kalra PS. Neuropeptide $Y$ secretion increases in the paraventricular nucleus in association with increased appetite for food. Proc Natl Acad Sci U S A. 1991;88(23):10931-10935.

59. Johnstone LE, Fong TM, Leng G. Neuronal activation in the hypothalamus and brainstem during feeding in rats. Cell Metab. 2006;4(4):313-321.

60. Mandelblat-Cerf Y, et al. Arcuate hypothalamic AgRP and putative POMC neurons show opposite changes in spiking across multiple timescales. Elife. 2015;4:e07122.

61. Nogueiras R, Williams LM, Dieguez C. Ghrelin: new molecular pathways modulating appetite and adiposity. Obes Facts. 2010;3(5):285-292.

62. Nakamura Y, Yanagawa Y, Morrison SF, Nakamura K. Medullary reticular neurons mediate neuropeptide Y-induced metabolic inhibition and mastication. Cell Metab. 2017;25(2):322-334.

63. Bruinstroop E, Fliers E, Kalsbeek A. Hypothalamic control of hepatic lipid metabolism via the autonomic nervous system. Best Pract Res Clin Endocrinol Metab. 2014;28(5):673-684.

64. Joly-Amado A, et al. The hypothalamic arcuate nucleus and the control of peripheral substrates. Best Pract Res Clin Endocrinol Metab. 2014;28(5):725-737.

65. Nogueiras R, et al. The central melanocortin system directly controls peripheral lipid metabolism. JClin Invest. 2007;117(11):3475-3488.

66. Bartness TJ, Liu Y, Shrestha YB, Ryu V. Neural innervation of white adipose tissue and the control of lipolysis. Front Neuroendocrinol. 2014;35(4):473-493.

67. Lambert GW, Straznicky NE, Lambert EA, Dixon JB, Schlaich MP. Sympathetic nervous activation in obesity and the metabolic syndrome--causes, consequences and therapeutic implications. Pharmacol Ther. 2010;126(2):159-172.

68. Kohsaka A, et al. High-fat diet disrupts behavioral and molecular circadian rhythms in mice. Cell Metab. 2007;6(5):414-421.

69. Hansen MJ, Jovanovska V, Morris MJ. Adaptive responses in hypothalamic neuropeptide $\mathrm{Y}$ in the face of prolonged high-fat feeding in the rat. J Neurochem. 2004;88(4):909-916.

70. Lin S, Storlien LH, Huang XF. Leptin receptor, NPY, POMC mRNA expression in the diet-induced obese mouse brain. Brain Res. 
2000;875(1-2):89-95.

71. la Fleur SE, van Rozen AJ, Luijendijk MC, Groeneweg F, Adan RA. A free-choice high-fat high-sugar diet induces changes in arcuate neuropeptide expression that support hyperphagia. Int JObes (Lond). 2010;34(3):537-546.

72. Lee AK, et al. Effect of high-fat feeding on expression of genes controlling availability of dopamine in mouse hypothalamus. Nutrition. 2010;26(4):411-422.

73. Zammaretti F, Panzica G, Eva C. Sex-dependent regulation of hypothalamic neuropeptide Y-Y1 receptor gene expression in moderate/high fat, high-energy diet-fed mice. J Physiol (Lond). 2007;583(Pt 2):445-454.

74. Mele P, Zammaretti F, Longo A, Panzica G,
Oberto A, Eva C. Sex-dependent regulation of hypothalamic neuropeptide Y-Y1 receptor gene expression in leptin treated obese (ob/ob) or lean mice. Brain Res. 2016;1649(Pt A):102-109.

75. Tong Q, Ye CP, Jones JE, Elmquist JK, Lowell BB. Synaptic release of GABA by AgRP neurons is required for normal regulation of energy balance. Nat Neurosci. 2008;11(9):998-1000.

76. van den Pol AN, et al. Neuromedin B and gastrinreleasing peptide excite arcuate nucleus neuropeptide $Y$ neurons in a novel transgenic mouse expressing strong Renilla green fluorescent protein in NPY neurons. J Neurosci. 2009;29(14):4622-4639.

77. Opland D, et al. Loss of neurotensin receptor-1 disrupts the control of the mesolimbic dopamine system by leptin and promotes hedonic feeding and obesity. Mol Metab. 2013;2(4):423-434.

78. Garfield AS, et al. A neural basis for melanocortin-4 receptor-regulated appetite. Nat Neurosci. 2015;18(6):863-871.

79. Harlan SM, Morgan DA, Dellsperger DJ, Myers MG, Mark AL, Rahmouni K. Cardiovascular and sympathetic effects of disrupting tyrosine 985 of the leptin receptor. Hypertension. 2011;57(3):627-632.

80. Paxinos G, Franklin KBJ. The mouse brain in stereotaxic coordinates. New York, New York, USA: Academic Press; 2001.

81. Madden CJ, Tupone D, Cano G, Morrison SF. $\alpha 2$ Adrenergic receptor-mediated inhibition of thermogenesis. JNeurosci. 2013;33(5):2017-2028. 